\title{
Detecting Quaternionic Maps Between Hyperkähler Manifolds
}

\author{
Jingyi Chen $\cdot$ Jiayu Li
}

Received: 18 July 2013 / Accepted: 19 September 2013 / Published online: 1 November 2013

(C) School of Mathematical Sciences, University of Science and Technology of China and Springer-Verlag Berlin Heidelberg 2013

\begin{abstract}
For a harmonic map between two hyperkäher manifolds, we prove a Weitzenböck type formula for the defining quantity of quaternionic maps, and apply it to harmonic morphisms. We also provide a sufficient and necessary condition for a smooth map being quaternionic.
\end{abstract}

Keywords Harmonic maps · Hyperkähler manifolds · Quaternionic maps · Weitzenböck formula

Mathematics Subject Classification (2010) 53C26 · 53C43

\section{Introduction}

A Riemannian manifold $(M, g)$ is called hyperkähler if it possesses covariant constant, in the covariant differentiation $\nabla_{g}$, complex structures $J^{1}, J^{2}, J^{3}$ which satisfy the quaternionic relation: $J^{3}=J^{1} J^{2}$. The covariant constant complex structures

J. Chen is partially supported by a NSERC grant.

$\mathrm{J}$. Li is partially supported by National Science Foundation of China.

J. Chen

Department of Mathematics, University of British Columbia, Vancouver, B.C. V6T 1Z2, Canada e-mail: jychen@math.ubc.ca

J. Li (凶)

School of Mathematical Sciences, University of Science and Technology of China, Hefei 230026, Anhui, People's Republic of China

e-mail: jiayuli@ustc.edu.cn

J. Li

Academy of Mathematics and Systems Science, Chinese Academy of Sciences, Beijing 100190,

People's Republic of China 
$a J^{1}+b J^{2}+c J^{3}$ with $(a, b, c) \in \mathbb{S}^{2}$ is referred to the hyperkähler $\mathbb{S}^{2}$. A hyperkähler manifold is Ricci-flat and its dimension is a multiple of 4.

Let $M$ and $N$ be two hyperkähler manifolds with complex structures $J^{Q}$ and $\mathcal{J}^{P}$, respectively, for $P, Q=1,2,3$ which satisfy the quaternionic relation. A smooth map $f: M \rightarrow N$ is called a quaternionic map if

$$
\sum_{P, Q} A_{P Q} \mathcal{J}^{P} \circ d f \circ J^{Q}=d f
$$

where $A_{P Q}$ denote the entries of a constant matrix $A$ in $S O(3)$. Arising from a dimension reduction procedure in higher dimensional gauge theory, these maps are nonlinear generalization of the Cauchy-Riemann-Fueter equations for functions between the quaternions, and they are called triholomorphic maps in [6]. It is known that a quaternionic map is harmonic, in fact, it minimizes the Dirichlet energy in its homotopy class when $M$ is compact [6] (also see [2, 9]). Also known is that a holomorphic map is quaternionic but the converse is not necessarily true since the rank of the differential of such a map can be odd [3]. In fact, there is a homotopy condition that determines whether a quaternionic map is holomorphic (in any complex structures in the hyperkähler $\mathbb{S}^{2}$ ) or not (see [3, Theorem 3.1]).

In this note, we explore various conditions which force harmonic maps or smooth maps to be quaternionic. The first result is a Weitzenböck type formula useful for detecting when a harmonic map is quaternionic.

Theorem 1.1 Let $M^{4 m}$ and $N^{4 n}$ be two hyperkähler manifolds with covariant constant complex structures $J^{1}, J^{2}, J^{3}=J^{1} J^{2}$ and $\mathcal{J}^{1}, \mathcal{J}^{2}, \mathcal{J}^{3}=\mathcal{J}^{1} \mathcal{J}^{2}$, respectively. Suppose that $f: M \rightarrow N$ is a harmonic map. Let $\phi=d f-\sum_{P, Q=1}^{3} A_{P Q} \mathcal{J}^{P} \circ d f \circ$ $J^{Q}$ where $\left(A_{P Q}\right) \in S O(3)$. Then

$$
\Delta|\phi|^{2}=2|\nabla \phi|^{2}-8 \sum_{i, j=1}^{4 m} R^{N}\left(\phi \cdot e_{i}, d f \cdot e_{j}, d f \cdot e_{i}, d f \cdot e_{j}\right)
$$

where $R^{N}$ is the curvature tensor of $N$ and $\left\{e_{1}, \ldots, e_{4 m}\right\}$ is an orthonormal basis at the point under consideration on $M$. Moreover, if $M$ is compact and $f$ is harmonic morphism, then $f$ satisfies (1.1) on $M$ if it does so at one point on $M$.

Here we recall that a map from a manifold to another is called a harmonic morphism if it preserves the Laplace equation, i.e., the composition of the map with any local harmonic function on the domain is harmonic on the target.

In the second result, we find a necessary and sufficient condition, in terms of quantities that depend only on homotopy classes of smooth maps from $M$ to $N$, for a smooth map being quaternionic.

Theorem 1.2 Suppose that $u: M \rightarrow N$ is a smooth map where $M, N$ are hyperkähler manifolds as above and $M$ is compact. Let $B$ be the matrix with entries

$$
B_{P Q}=-\int_{M}\left\langle J^{P}, u^{*} \mathcal{J}^{Q}\right\rangle d V \quad \text { for } P, Q=1,2,3 \text {. }
$$


Then $u$ is a quaternionic map if and only if

$$
\operatorname{det} B \geq 0 \quad \text { and } \quad \operatorname{tr} \sqrt{B B^{t}}=E(u)
$$

where $E(u)$ is the Dirichlet energy of $u$.

Using this criterion we can easily determine which homotopy class of differentiable maps from a flat torus $\mathbb{T}^{4 m}$ to $\mathbb{T}^{4 n}$ contains a quaternionic representative.

Throughout this note, $M, N$ are hyperkähler manifolds of dimension $4 m, 4 n$, with $J^{1}, J^{2}, J^{3}$ and $\mathcal{J}^{1}, \mathcal{J}^{2}, \mathcal{J}^{3}$ which satisfy the quaternionic relation, respectively. The following ranges for indices are adapted:

$$
\begin{aligned}
& 1 \leq i, j, k, l \leq 4 m, \\
& 1 \leq \alpha, \beta, \gamma, \delta, \eta \leq 4 n, \\
& 1 \leq P, Q, S, T, U, V \leq 3 .
\end{aligned}
$$

Summation is taken over indices according to their appropriate range.

\section{A Weitzenböck Formula for Harmonic Maps}

In this section, we will prove Theorem 1.1.

In local coordinates, we write $f_{i}^{\alpha}=\partial f^{\alpha} / \partial x^{i}$ and the complex structures

$$
J^{P} \frac{\partial}{\partial x^{k}}=\sum_{i}\left(J^{P}\right)_{k}{ }^{i} \frac{\partial}{\partial x^{i}} \quad \text { and } \quad \mathcal{J}^{P} \frac{\partial}{\partial y^{\alpha}}=\sum_{\beta}\left(\mathcal{J}^{P}\right)_{\alpha}{ }^{\beta} \frac{\partial}{\partial y^{\beta}} .
$$

It follows that

$$
\sum_{l}\left(J^{P}\right)_{j}{ }^{l} g_{k l}=\left\langle\frac{\partial}{\partial x^{k}}, J^{P} \frac{\partial}{\partial x^{j}}\right\rangle=-\left\langle J^{P} \frac{\partial}{\partial x^{k}}, \frac{\partial}{\partial x^{j}}\right\rangle=-\sum_{i}\left(J^{P}\right)_{k}{ }^{i} g_{i j} .
$$

Equation (1.1) reads

$$
\sum_{j, \beta} A_{P Q}\left(J^{P}\right)_{i}{ }^{j}\left(\mathcal{J}^{Q}\right)_{\beta}{ }^{\alpha} f_{j}^{\beta}=f_{i}^{\alpha}
$$

We introduce

$$
\phi=d f-\sum_{P, Q} A_{P Q} \mathcal{J}^{P} \circ d f \circ J^{Q}
$$


Proof of Theorem 1.1 We calculate by using the moving frames as in [4, (1.11)]:

$$
\begin{aligned}
f_{i j j}^{\alpha} & =f_{j i j}^{\alpha} \\
& =f_{j j i}^{\alpha}+\sum_{k} f_{k}^{\alpha} R_{k j i j}^{M}-\sum_{\beta, \gamma, \delta} f_{j}^{\beta} f_{i}^{\gamma} f_{j}^{\delta} R_{\alpha \beta \gamma \delta}^{N} \\
& =f_{j j i}^{\alpha}+\sum_{k} f_{k}^{\alpha} R i c_{k i}^{M}-\sum_{\beta, \gamma, \delta} f_{j}^{\beta} f_{i}^{\gamma} f_{j}^{\delta} R_{\alpha \beta \gamma \delta}^{N},
\end{aligned}
$$

where $R_{\alpha \beta \gamma \eta}^{N}$ is the curvature tensor of $N$ and $R i c_{k i}^{M}$ is the Ricci tensor of $M$. Because $M$ is Ricci-flat and $f$ is harmonic, we have

$$
f_{i j j}^{\alpha}=-\sum_{\beta, \gamma, \delta} f_{j}^{\beta} f_{i}^{\gamma} f_{j}^{\delta} R_{\alpha \beta \gamma \delta}^{N}
$$

Since matrices in $S O(3)$ preserves the quaternionic relation, namely, if $J^{3}=J^{1} J^{2}$ and $A \in S O(3)$, then $A\left(J^{3}\right)=A\left(J^{1}\right) A\left(J^{2}\right)$. We may therefore assume, for simplicity, that the matrix $\left(A_{P Q}\right)$ in $(1.1)$ is the identity matrix $I_{3 \times 3}$. In the local coordinates, we write

$$
\phi_{i}^{\alpha}=f_{i}^{\alpha}-\sum_{k, \beta, P}\left(\mathcal{J}^{P}\right)_{\beta}{ }^{\alpha} f_{k}^{\beta}\left(J^{P}\right)_{i}{ }^{k} .
$$

From (2.3) and that the complex structures are covariant constant, it follows that

$$
\begin{aligned}
\phi_{i j j}^{\alpha} & =f_{i j j}^{\alpha}-\sum_{k, \beta, P}\left(\mathcal{J}^{P}\right)_{\beta}{ }^{\alpha} f_{k j j}^{\beta}\left(J^{P}\right)_{i}{ }^{k} \\
& =-\sum_{\beta, \gamma, \delta} f_{j}^{\beta} f_{i}^{\gamma} f_{j}^{\delta} R_{\alpha \beta \gamma \delta}^{N}+\sum_{k, \beta, \gamma, \delta, \eta, P}\left(\mathcal{J}^{P}\right)_{\beta}{ }^{\alpha} f_{j}^{\gamma} f_{k}^{\delta} f_{j}^{\eta} R_{\beta \gamma \delta \eta}^{N}\left(J^{P}\right)_{i}{ }^{k} .
\end{aligned}
$$

We set

$$
\psi_{i}^{\alpha}=\sum_{k, \beta, P}\left(\mathcal{J}^{P}\right)_{\beta}{ }^{\alpha} f_{k}^{\beta}\left(J^{P}\right)_{i}{ }^{k} .
$$

Then we have

$$
\begin{aligned}
\sum_{i, j, \alpha} \phi_{i}^{\alpha} \phi_{i j j}^{\alpha}= & \sum_{i, j, \alpha}\left(f_{i}^{\alpha}-\sum_{k, \beta, P}\left(\mathcal{J}^{P}\right)_{\beta}{ }^{\alpha} f_{k}^{\beta}\left(J^{P}\right)_{i}{ }^{k}\right)\left(-\sum_{\beta, \gamma, \delta} f_{j}^{\beta} f_{i}^{\gamma} f_{j}^{\delta} R_{\alpha \beta \gamma \delta}^{N}\right. \\
& \left.+\sum_{k, \beta, \gamma, \delta, \eta, P}\left(\mathcal{J}^{P}\right)_{\beta}{ }^{\alpha} f_{j}^{\gamma} f_{k}^{\delta} f_{j}^{\eta} R_{\beta \gamma \delta \eta}^{N}\left(J^{P}\right)_{i}{ }^{k}\right) \\
= & -\sum_{i, j, \alpha, \beta, \gamma, \delta} f_{i}^{\alpha} f_{j}^{\beta} f_{i}^{\gamma} f_{j}^{\delta} R_{\alpha \beta \gamma \delta}^{N}+\sum_{i, j, \alpha, \beta, \gamma, \delta} \psi_{i}^{\alpha} f_{j}^{\beta} f_{i}^{\gamma} f_{j}^{\delta} R_{\alpha \beta \gamma \delta}^{N}
\end{aligned}
$$




$$
\begin{aligned}
& +\sum_{i, j, k, \alpha, \beta, \gamma, \delta, \eta, P} f_{i}^{\alpha} f_{j}^{\gamma} f_{k}^{\delta} f_{j}^{\eta}\left(\mathcal{J}^{P}\right)_{\beta}{ }^{\alpha}\left(J^{P}\right)_{i}{ }^{k} R_{\beta \gamma \delta \eta}^{N} \\
& -\sum_{i, j, k, \alpha, \beta, \gamma, \delta, \eta, P} f_{j}^{\gamma} f_{k}^{\delta} f_{j}^{\eta} \psi_{i}^{\alpha}\left(\mathcal{J}^{P}\right)_{\beta}{ }^{\alpha}\left(J^{P}\right)_{i}{ }^{k} R_{\beta \gamma \delta \eta}^{N} .
\end{aligned}
$$

In orthonormal frames,

$$
\left(J^{P}\right)_{k}{ }^{i}=-\left(J^{A}\right)_{i}{ }^{k} \quad \text { and } \quad\left(\mathcal{J}^{P}\right)_{\alpha}{ }^{\beta}=-\left(\mathcal{J}^{P}\right)_{\beta}{ }^{\alpha}
$$

We have

$$
\begin{aligned}
& \sum_{i, j, k, \alpha, \beta, \gamma, \delta, \eta, P} f_{i}^{\alpha} f_{j}^{\gamma} f_{k}^{\delta} f_{j}^{\eta}\left(\mathcal{J}^{P}\right)_{\beta}{ }^{\alpha}\left(J^{P}\right)_{i}{ }^{k} R_{\beta \gamma \delta \eta}^{N} \\
= & \sum_{i, j, k, \alpha, \beta, \gamma, \delta, \eta, P} f_{i}^{\alpha} f_{j}^{\gamma} f_{k}^{\delta} f_{j}^{\eta}\left(-\mathcal{J}^{P}\right)_{\alpha}{ }^{\beta}\left(-J^{P}\right)_{k}{ }^{i} R_{\beta \gamma \delta \eta}^{N} \\
= & \sum_{j, k, \beta, \gamma, \delta \eta} f_{j}^{\gamma} f_{k}^{\delta} f_{j}^{\eta} \psi_{k}^{\beta} R_{\beta \gamma \delta \eta}^{N} .
\end{aligned}
$$

Furthermore, when $P \neq Q$,

$$
\begin{aligned}
\sum_{i, \alpha, P, Q}\left(\mathcal{J}^{Q}\right)_{\tau}{ }^{\alpha}\left(\mathcal{J}^{P}\right)_{\beta}{ }^{\alpha}\left(J^{Q}\right)_{i}{ }^{l}\left(J^{P}\right)_{i}{ }^{k} & =\sum_{i, \alpha, P, Q}\left(\mathcal{J}^{Q}\right)_{\tau}{ }^{\alpha}\left(-\mathcal{J}^{P}\right)_{\alpha}{ }^{\beta}\left(J^{Q}\right)_{i}{ }^{l}\left(-J^{P}\right)_{k}{ }^{i} \\
& =\sum_{i, \alpha, P, Q}\left(\mathcal{J}^{Q}\right)_{\tau}{ }^{\alpha}\left(\mathcal{J}^{P}\right)_{\alpha}{ }^{\beta}\left(J^{P}\right)_{k}{ }^{i}\left(J^{Q}\right)_{i}{ }^{l} \\
& =\sum_{S}\left(\mathcal{J}^{S}\right)_{\tau}{ }^{\beta}\left(-J^{S}\right)_{k}{ }^{l} \\
& =-\sum_{S}\left(\mathcal{J}^{S}\right)_{\tau}{ }^{\beta}\left(J^{S}\right)_{k}{ }^{l}
\end{aligned}
$$

where $S \neq P, Q$ and we have used the quaternionic relation for the two triples of complex structures. By grouping the terms with $P=Q$ together and using (2.6) for the rest, we have

$$
\begin{aligned}
& \sum_{i, j, k, \alpha, \beta, \gamma, \delta, \eta, P} f_{j}^{\gamma} f_{k}^{\delta} f_{j}^{\eta} \psi_{i}^{\alpha}\left(\mathcal{J}^{P}\right)_{\beta}{ }^{\alpha}\left(J^{P}\right)_{i}{ }^{k} R_{\beta \gamma \delta \eta}^{N} \\
= & \sum_{i, j, k, \alpha, \beta, \gamma, \delta, \eta} f_{j}^{\gamma} f_{k}^{\delta} f_{j}^{\eta}\left\{\sum_{l, \tau, Q}\left(\mathcal{J}^{Q}\right)_{\tau}{ }^{\alpha} f_{l}^{\tau}\left(J^{Q}\right)_{i}{ }^{l}\right\}\left(\mathcal{J}^{P}\right)_{\beta}{ }^{\alpha}\left(J^{P}\right)_{i}{ }^{k} R_{\beta \gamma \delta \eta}^{N} \\
= & 3 \sum_{j, k, \beta, \gamma, \delta, \eta} f_{j}^{\gamma} f_{k}^{\delta} f_{j}^{\eta} f_{k}^{\beta} R_{\beta \gamma \delta \eta}^{N}
\end{aligned}
$$




$$
\begin{aligned}
& -2 \sum_{j, k, l, \beta, \gamma, \delta, \eta, \tau, Q} f_{j}^{\gamma} f_{k}^{\delta} f_{j}^{\eta}\left(\mathcal{J}^{Q}\right)_{\tau}{ }^{\beta} f_{l}^{\tau}\left(J^{Q}\right)_{k}{ }^{l} R_{\beta \gamma \delta \eta}^{N} \\
= & 3 \sum_{j, k, \beta, \gamma, \delta, \eta} f_{j}^{\gamma} f_{k}^{\delta} f_{j}^{\eta} f_{k}^{\beta} R_{\beta \gamma \delta \eta}^{N}-2 \sum_{j, k, \beta, \gamma, \delta, \eta} f_{j}^{\gamma} f_{k}^{\delta} f_{j}^{\eta} \psi_{k}{ }^{\beta} R_{\beta \gamma \delta \eta}^{N} .
\end{aligned}
$$

Insert (2.5), (2.7) into (2.4):

$$
\begin{aligned}
\sum_{i, j, \alpha} \phi_{i}^{\alpha} \phi_{i j j}^{\alpha} & =-4 \sum_{i, j, \beta, \gamma, \delta, \eta} f_{i}^{\gamma} f_{i}^{\delta} f_{j}^{\beta} f_{j}^{\eta} R_{\gamma \beta \delta \eta}^{N}+4 \sum_{i, j, \beta, \gamma, \delta, \eta} \psi_{i}^{\gamma} f_{i}^{\delta} f_{j}^{\beta} f_{j}^{\eta} R_{\gamma \beta \delta \eta}^{N} \\
& =-4 \sum_{i, j, \beta, \gamma, \delta, \eta} \phi_{i}^{\gamma} f_{i}^{\delta} f_{j}^{\beta} f_{j}^{\eta} R_{\gamma \beta \delta \eta}^{N} .
\end{aligned}
$$

This completes the proof of the first part of the theorem.

Now assume $f$ is a harmonic morphism. A result in [7] and [8] asserts that $f$ is harmonic and weakly horizontal conformal, where the latter means: for each $p \in M$, either $d f_{p}=0$ or $d f_{p}$ maps the horizontal space $\left(\operatorname{ker} d f_{p}\right)^{\perp}$ conformally onto $T_{f(p)} N$, i.e., $d f_{p}$ is surjective and there exists a number $\kappa(p) \neq 0$ such that

$$
h\left(d f_{p}(X), d f_{p}(Y)\right)=\kappa(p)^{2} g(X, Y), \quad \forall X, Y \in\left(\operatorname{ker} d f_{p}\right)^{\perp} .
$$

Then at any point $p \in M$, either $|\nabla f|^{2}(p)=0$ hence $R^{N}\left(\phi \cdot e_{i}, d f \cdot e_{j}, d f \cdot e_{i}\right.$, $\left.d f \cdot e_{j}\right)=0$, or there is an orthonormal set of tangent vectors $e_{1}, \ldots, e_{4 n} \in\left(\operatorname{ker} d f_{p}\right)^{\perp}$ such that

$$
d f \cdot e_{i}=|\nabla f|^{2} w_{i}
$$

form an orthonormal basis for $T_{f(p)} N$. In this case,

$$
\sum_{j} R^{N}\left(\phi \cdot e_{i}, d f \cdot e_{j}, d f \cdot e_{i}, d f \cdot e_{j}\right)=|\nabla f|^{2} \operatorname{Ric}^{N}\left(\phi \cdot e_{i}, d f \cdot e_{i}\right)=0
$$

as $N$ is Ricci-flat. Therefore,

$$
\Delta|\phi|^{2}=2|\nabla \phi|^{2}
$$

When $M$ is compact, the subharmonic function $|\phi|^{2}$ is constant, hence vanishes identically on $M$ if it does so at one point.

By a similar argument, we can show

Corollary 2.1 Let $M$ and $N$ be two hyperkähler manifolds. Assume that $M$ is compact and $N$ is flat. Then $f$ is a quaternionic map if it satisfies (1.1) at one point on $M$.

\section{A Homotopy Condition for Smooth Mappings Being Quaternionic}

In this section, we will prove Theorem 1.2. 
The energy functional on $C^{\infty}(M, N)$ is

$$
E(u)=\frac{1}{2}\|d u\|^{2}=\frac{1}{2} \int_{M} \sum_{i, j, \alpha, \beta} g^{i j} h_{\alpha \beta} \partial_{i} u^{\alpha} \partial_{j} u^{\beta} d V
$$

where $g, h$ are the Riemannian metrics on $M, N$ respectively and $d V$ is the volume element on $M$. Define a functional $E_{T}: C^{\infty}(M, N) \times S O(3) \rightarrow \mathbb{R}$ by

$$
E_{T}(u, A)=\sum_{P, Q} A_{P Q} \int_{M}\left\langle J^{P}, u^{*} \mathcal{J}^{Q}\right\rangle d V .
$$

Fix two sets of complex structures $J^{1}, J^{2}, J^{3}=J^{1} J^{2}$ on $M$ and $\mathcal{J}^{1}, \mathcal{J}^{2}, \mathcal{J}^{3}=$ $\mathcal{J}^{1} \mathcal{J}^{2}$ on $N$ and define a functional $I: C^{\infty}(M, N) \times S O(3) \rightarrow \mathbb{R}$ by

$$
I(u, A)=\frac{1}{2} \int_{M}\left|d u-\sum_{P, Q} A_{P Q} \mathcal{J}^{P} \circ d u \circ J Q\right|^{2} d V .
$$

It is clear that $I(u)=0$ if and only if $u$ is a quaternionic map.

Each $J^{P}$ or $\mathcal{J}^{Q}$ determines a Kähler form on $M$ or $N$, and it is well-known $[1,5]$ that $\int_{M}\left\langle J^{P}, u^{*} \mathcal{J}^{Q}\right\rangle d V$ only depends on the homotopy class of $u$. Hence, for any given $A \in S O(3), E_{T}(u, A)$ is homotopy invariant which means that, if $u$ is homotopy to $v$ then $E_{T}(u, A)=E_{T}(v, A)$. The following proposition is first proved in [6], see also [2] and [9].

Proposition 3.1 For any smooth map $u: M \rightarrow N$ and $A \in S O(3)$, we have

$$
E(u)+E_{T}(u, A)=\frac{1}{4} I(u, A) .
$$

If $u$ is a quaternionic map, then it minimizes energy in its homotopy class.

Now we are ready to prove Theorem 1.2.

Proof of Theorem 1.2 By Proposition 3.1 and $I(u, A) \geq 0$ for all $A \in S O(3)$, it is clear that if $u$ solves the quaternionic equation (1.1) with $A \in S O(3)$, then

$$
\begin{aligned}
E(u) & =\min _{A \in S O(3)}-\sum_{P, Q} A_{P Q} \int_{M}\left\langle J^{P}, u^{*} \mathcal{J}^{Q}\right\rangle d V \\
& =\min _{A \in S O(3)} \sum_{P, Q} A_{P Q} B_{P Q} \\
& =\min _{A \in S O(3)} \operatorname{tr} A B^{t} .
\end{aligned}
$$

For the constraint $A \in S O(3)$, the Lagrange multiplier method yields: for extreme values

$$
\frac{\partial}{\partial A_{P Q}}\left(\sum_{S, T} A_{S T} B_{S T}+\sum_{S, T} \lambda_{S T}\left(\delta_{S T}-A_{S K} A_{T K}\right)\right)=0
$$


and for local minima

$$
\frac{\partial^{2}}{\partial A_{P Q} \partial A_{U V}}\left(\sum_{S, T} A_{S T} B_{S T}+\sum_{S, T} \lambda_{S T}\left(\delta_{S T}-\sum_{K} A_{S K} A_{T K}\right)\right) \geq 0 .
$$

By (3.2), we get

$$
B_{P Q}=\sum_{K} \lambda_{P K} A_{K Q}+\sum_{K} \lambda_{K P} A_{K Q} .
$$

Let $\lambda$ be the $3 \times 3$ matrix with entries $\lambda_{P Q}$ and let $\Lambda=\lambda+\lambda^{t}$. The last identity can be rewritten as

$$
B=\Lambda A \text {. }
$$

The inequality (3.3) implies that $\Lambda$ is nonnegative definite, in turn, it implies that $\operatorname{det} B \geq 0$. In summary, $u$ is a quaternionic map only if

$$
\left\{\begin{array}{l}
\Lambda A=B \\
\operatorname{tr} \Lambda=E(u) \\
\operatorname{det} B \geq 0
\end{array}\right.
$$

It follows that for a quaternionic map $u$, we have by (3.4)

$$
B B^{t}=\Lambda A A^{t} \Lambda=\Lambda^{2},
$$

therefore,

$$
\operatorname{tr} \sqrt{B B^{t}}=\operatorname{tr} \Lambda=E(u) .
$$

We have shown that a quaternionic map satisfies (1.2).

Conversely, assume (1.2) holds for a smooth map $u$. There exists $S \in S O$ (3) diagonalizing the symmetric matrix

$$
B B^{t}=S^{t}\left(\begin{array}{ccc}
\mu_{1} & 0 & 0 \\
0 & \mu_{2} & 0 \\
0 & 0 & \mu_{3}
\end{array}\right) S
$$

where $\mu_{1}, \mu_{2}, \mu_{3}$ are nonnegative since $B B^{t}$ is semi-positive definite. Then define

$$
\Lambda=S^{t}\left(\begin{array}{ccc}
\sqrt{\mu_{1}} & 0 & 0 \\
0 & \sqrt{\mu_{2}} & 0 \\
0 & 0 & \sqrt{\mu_{3}}
\end{array}\right) S .
$$

We claim that there exists $A \in S O(3)$ which solves the equation

$$
\Lambda A=B .
$$

To see this, we consider two cases. 
First, assume det $\Lambda \neq 0$. Take $A=\Lambda^{-1} B$ and we check directly from (3.5) and (3.6) that $A \in S O(3): A A^{t}=\Lambda^{-1} B B^{t}\left(\Lambda^{-1}\right)^{t}=I_{3 \times 3}$ and $\operatorname{det} A>0$ since $\operatorname{det} B>0$ and $\operatorname{det} \Lambda>0$.

Second, assume $\operatorname{det} \Lambda=0$. There are three cases.

Case (i): $\mu_{3}=0, \mu_{1} \neq 0, \mu_{2} \neq 0$. Equation (3.7) is equivalent to

$$
\left(\begin{array}{ccc}
\sqrt{\mu_{1}} & 0 & 0 \\
0 & \sqrt{\mu_{2}} & 0 \\
0 & 0 & 0
\end{array}\right) S A=S B .
$$

Set $S B=\left(C_{P Q}\right)_{3 \times 3}$. By (3.5) and $\mu_{3}=0$, we have

$$
C_{3 P}=0
$$

and

$$
C C^{t}=S B B^{t} S^{t}=\left(\begin{array}{ccc}
\mu_{1} & 0 & 0 \\
0 & \mu_{2} & 0 \\
0 & 0 & 0
\end{array}\right) .
$$

We then define

$$
D_{1 P}=\frac{1}{\sqrt{\mu_{1}}} C_{1 P} \quad \text { and } \quad D_{2 P}=\frac{1}{\sqrt{\mu_{2}}} C_{2 P},
$$

and it follows from (3.10) that $\left(D_{11}, D_{12}, D_{13}\right),\left(D_{21}, D_{22}, D_{23}\right)$ are mutually orthogonal unit vectors and we can choose $D_{3 P}$ as the third row together with $D_{1 P}, D_{2 P}$ to form a matrix $D$ in $S O$ (3). It then follows from (3.9) that $S A=D$ solves (3.8).

Case (ii): $\mu_{1} \neq 0, \mu_{2}=\mu_{3}=0$. Observe (3.8) is further simplified by setting $\mu_{2}=0$, and consequently $C_{2 P}=C_{3 P}=0$ and we can choose $D_{2 P}, D_{3 P}$, together with $D_{1 P}=C_{1 P} / \sqrt{\mu_{1}}$ to form $D \in S O(3)$ and then solve (3.8) by $S A=D$.

Case (iii): $\mu_{1}=\mu_{2}=\mu_{3}=0$. In this case, $\Lambda=B=0$ and any $A$ solves (3.7).

Therefore, we have shown that the above claim holds.

The solvability of (3.7) and the equality in the assumption (1.2) assert

$$
\begin{aligned}
E_{T}(u, A) & =-\sum_{P, Q} A_{P Q} B_{P Q} \\
& =-\sum_{P, Q}\left(A^{t}\right)_{Q P} B_{P Q} \\
& =-\sum_{P<Q}\left(A^{-1}\right)_{Q P} B_{P Q} \\
& =-\sum_{P} \Lambda_{P P} \\
& =-\operatorname{tr} \Lambda \\
& =-E(u) .
\end{aligned}
$$


Now it follows from Proposition 3.1 that $I(u, A)=0$, hence $u$ is a solution to the quaternionic equation (1.1) with the above $A$.

We recall the following criterion [3] which detects when a quaternionic map becomes holomorphic with respect to some complex structures in the hyperkähler $\mathbb{S}^{2}$.

Theorem 3.1 Suppose u is a quaternionic map. Let B be as in Theorem 1.2. Then

$$
(\operatorname{tr} B)^{2} \geq \max \left\{\text { eigenvalues of } B B^{t}\right\}
$$

and the equality holds if and only if $u$ is a holomorphic map with respect to some complex structures in the hyperkähler $\mathbb{S}^{2}$ on $M$ and on $N$.

Combining Theorems 1.2 and 3.1, we obtain a homotopy condition which detects which homotopy class admits a holomorphic map between hyperkähler manifolds.

\section{References}

1. Berger, M.: Sur les groupes d'holonomie des variété à connexion affine et variété riemanniennes. Bull. Soc. Math. Fr. 83, 279-330 (1955)

2. Chen, J.: Complex anti-self-dual connections on product of Calabi-Yau surfaces and triholomorphic curves. Commun. Math. Phys. 201, 201-247 (1999)

3. Chen, J., Li, J.: Quaternionic maps between hyperkähler manifolds. J. Differ. Geom. 55, 355-384 (2000)

4. Cheng, S.Y.: Liouville theorem for harmonic maps. Proc. Symp. Pure Math. 36, 147-151 (1980)

5. Eells, J., Lemaire, L.: Selected Topics in Harmonic Maps. C.B.M.S. Regional Conf. Series, vol. 50. Am. Math. Soc., Providence (1983)

6. Figuroa-O'Farrill, J.M., Köhl, C., Spence, B.: Supersymmetric Yang-Mills, octonionic instantons and triholomorphic curves. Nucl. Phys. B 521(3), 419-443 (1998)

7. Fuglede, B.: Harmonic morphisms between Riemannian manifolds. Ann. Inst. Fourier (Grenoble) 28(2), 107-144 (1978)

8. Ishihara, T.: A mapping of Riemannian manifolds which preserves harmonic functions. J. Math. Kyoto Univ. 19, 215-229 (1979)

9. Li, J., Tian, G.: A blow-up formula for stationary harmonic maps. Int. Math. Res. Not. 14, 735-755 (1998) 